\title{
Bitcoin daily close price prediction using optimized grid search method
}

\author{
Marzieh ROSTAMI \\ Department of Information Technology \\ Engineering, \\ Raja University of Qazvin, Qazvin, Iran \\ email: mrz.rst@gmail.com
}

\author{
Mahdi BAHAGHIGHAT ${ }^{1}$ \\ Department of Computer Engineering, \\ Imam Khomeini International \\ University, Qazvin, Iran \\ email: Bahaghighat@eng.ikiu.ac.ir
}

\author{
Morteza MOHAMMADI \\ ZANJIREH \\ Department of Computer Engineering, \\ Imam Khomeini International \\ University, Qazvin, Iran \\ email: Zanjireh@eng.ikiu.ac.ir
}

\begin{abstract}
Cryptocurrencies are digital assets that can be stored and transferred electronically. Bitcoin (BTC) is one of the most popular cryptocurrencies that has attracted many attentions. The BTC price is considered as a high volatility time series with non-stationary and non-linear behavior. Therefore, the BTC price forecasting is a new, challenging, and open problem. In this research, we aim the predicting price using machine learning and statistical techniques. We deploy several robust approaches such as the Box-Jenkins, Autoregression (AR), Moving Average (MA), ARIMA, Autocorrelation Function (ACF), Partial Autocorrelation Function (PACF), and Grid Search algorithms to predict BTC price. To evaluate the performance of the proposed model, Forecast Error (FE), Mean
\end{abstract}

Computing Classification System 1998: G.2.2

Mathematics Subject Classification 2010: 68R15

Key words and phrases: bitcoin; cryptocurrency; price prediction; autoregression (AR); moving average (MA), ARIMA

${ }^{1}$ Corresponding author 
Forecast Error (MFE), Mean Absolute Error (MAE), Mean Squared Error (MSE), as well as Root Mean Squared Error (RMSE), are considered in our study.

\section{Introduction}

Fiat currencies are currently used to exchange daily payments but the exponential growth of the cryptocurrency market is a phenomenon that has got much attention in recent years. It is a new emerging financial ecosystem, so its opportunities and threats are under evaluation in many academic studies. There are some critical issues that should be analyzed, and the primary question is that whether the price dynamic behavior is predictable or not? Given the efficient market hypothesis (EMH), they have non-deterministic variation patterns, and Bitcoin (BTC) price should be assumed as a stochastic signal. BTC was created and introduced to the world as the first cryptocurrency coin , but since then, many other coins/tokens, so called Altcoins, have generated [12]. Today, about 10,000 coins/tokens are actively traded, and the market capitalization increases noticeably. Many of them have different key features and applications. The various researches were conducted to answer whether BTC is a real currency or not? [43, 42]. Now, between three to six million investors in the private sector including institutions, and individuals (retailers) actively exchange different coins and tokens via well-known available trading networks [27]. In the second quartile (Q2) of 2017, the value of the available cryptocurrencies market, so-called Market Cap, exceeded 91 billion USD [19].

BTC is currently dominant in the market, its position as a strong leader (the king) is vulnerable due to technical issues of the first generation blockchain (G1), security, and the technological advances of new generations of cryptocurrencies (G2 and G3) [33, 13, 21]. Despite the relatively stable economic and gradual growing of interest in cryptocurrencies [3, 40, 14, 38], there is still no comprehensive analysis of cryptocurrency dynamics and ecosystems. In the research field, most of the existing studies focus on Bitcoin itself $[34,30]$, transaction network, BTC price behavior [18], BTC market trend, BTC dominance, regarding to a limited numbers of fiat currencies (in particular USD) [22, 21]. For example, there is even a disagreement about whether the dominant position of BTC may be compromised or not? Because, BTC dominance plays key

role in the whole ecosystem [22]. BTC is the most famous and pioneer coin; however, recent studies on the BTC total market share and other altcoins (in 
particular Ethereum (ETH)) indicate that its first rank would be in a real danger in the future.

BTC is an emerging digital currency with the very high volatility. In comparison to the legacy moneys, it leads to more complex challenges in the price prediction problem [11]. A lot of researches have been conducted for the traditional stock markets such as Nasdaq, Japan Exchange Group, New York Stock Exchange, etc. [28, 41], while there are a few studies for cryptocurrencies. It opened a new challenging problem in parallel. BTC price is a time series with a very high volatility and its forecasting problem is still in its early stage of gradual developments. Traditional methods for time series forecasting, such as Holt-Winters exponential models, basically assumes the linear behavior and needs data that can be divided into a trend, seasonal, and noise [15]. On the other hand, there are some approaches to predict BTC price based on Natural Language Processing (NLP) and sentiment analysis. Today, NLP as an AI (artificial intelligence) technology and Deep learning $[9,2]$ are used together in advanced text mining/analytic tools [23, 4, 26, 8, 7]. These approaches get social media text data from Twitter, Facebook, and etc., as the input and try to draw a link between the content of daily messages and the BTC price. These approaches are on their primal development steps now. The performance of Sentiment analysis are usually restricted to detect just big movements due to some important affecting news. In regular conditions, their performance degrades.

In this research, we aimed to predict the BTC price found on several different algorithms. The Box-Jenkins method, Autoregression, Autocorrelation and Partial Autocorrelation methods, ARIMA method, the Moving Average and Grid search approach are considered. In addition, the Forecast Error (FE), Mean Forecast Error (MFE), Mean Absolute Error (MAE), Mean Squared Error (MSE), as well as Root Mean Squared Error (RMSE), have been used to evaluate our proposed model.

The article is presented as follows: In Section 2, related works are mentioned, while in Section 3, the methodology of the current study is described. Section 4 also presents the simulation results. Finally, Section 5 summarizes and concludes this study.

\section{Related works}

In [32], the BTC price prediction was introduced using decision tree and regression techniques. The main idea was to get order book data and transfer them 
into features over time. These features were referred as feature series which can be used to make prediction models with emphasizing on both volatility and feature series. Their method depends on local data of a special broker/exchanger so the scalability issue should be improved in the future works.

[5] proposed a new approach found on computational intelligence. It used a hybrid controller based on the Neuro-Fuzzy so-called PATIOS to predict the BTC daily price. The results of their work show that their proposed method outperforms the simple neuro-fuzzy approach or simple model of artificial neural networks. The research demonstrated the use of the closed-loop or feedback control technique to expand the BTC and fuzzy modeling literature and uncertainty related to the dynamic behavior of BTC prices to overcome and gain a relatively positive return. PATIOS does not have a user-friendly interface. To enhance it, more focus on creating a UI/UX interface is essential.

In [24], a wide-scale vector model has been proposed to explain how price information was transferred among different crypto market brokers, exchanges, and between traditional markets and crypto ones. Accordingly, they introduced a Vector Autoregressive model (VAR). Their empirical findings suggest that there is a robust correlation between the prices of BTC in different cryptocurrency markets. In contrast, BTC-price correlation with most traditional assets is relatively low. This model can also improve the BTC price forecasts concerning a simple autoregressive model.

In [39], they compared the volatility of one stage and BTC-VaR (BTC value-at-risk $(\mathrm{VaR})$ ) forecasting using some important volatility models. It also considered methods that actually involve the presence of outdated data and strongly estimate fluctuations and VaR. The achieved results explicitly suggested that noises and outliers can play an influential role in modeling and forecasting BTC-VaR.

In [29], some graph models had been investigated to analyze BTC price fluctuations. It was found that the optimal model was AR-CGARCH in terms of a good fit with the data. The result indicated well the importance of the existence of both short-term and long-term components for an accurate prediction.

Machine learning (ML) is known as a sub-domain of data science. It can improve software applications to get more accurate results in various applications $[6,35,36,25,10]$. It deploys historical perceptions (data) as input to make new predictions. [31] proposed classifier and regression models based on machine learning with high performance for both mid-term and short-term long lines. In their work, the prediction was not restricted to just daily data. They extended the work for monthly and yearly data as well. The classification 
model can achieve above $65 \%$ accuracy for the next day price prediction and $64 \%$ to $62 \%$ accuracy for the 7 and 17 days forecasts. For the daily, the error rate is as low as $1.44 \%$, while for the seven to ninety-day horizons, it varies from $2.88 \%$ to $4.10 \%$.

To predict BTC price value at different frequencies using machine learning techniques, [17] first classified the price of BTC based on the price of the day and the high frequencies. A set of high- dimensional features include property, gold spot price, network, and trading market were used to predict BTC price on a daily time scale. Statistical methods (SM) include logistic regression (LR) along with linear analysis, were used to predict the daily price prediction with accuracy $66 \%$ accuracy, and more complex algorithms pass machine learning (ML). In comparison with results of the criterion for daily price prediction, the results are higher in SM and ML algorithms have $66 \%$ and $65.3 \%$ better performance, respectively. The machine learning models, such as Support Vector Machine (SVM), XGBoost, Random Forest (RF), and Quadratic Discriminant Analysis (QDA), were superior to SM for the 5 minutes price prediction (5m) with an accuracy of $67.2 \%$.

In the next section, we are going to discuss about methodology and the proposed model.

\section{Methodology}

In this section, our proposed model is proposed to provide a detailed forecast of price. For this purpose, first, the datasets used are introduced, and then the performance evaluation criteria and our proposed forecasting models such as Box-Jenkins method, ACF, PACF, ARIMA, MA, AR, and Grid Search are investigated.

\subsection{Dataset}

We have used the BTC price dataset from [44]. It provides price values from 1 December 2014 to 29 may 2020. The data is available in the daily time scale. The statistical summary of the dataset is presented in Table 1.

\begin{tabular}{c|c|c|c|c|c|c|c}
\hline count & mean & std & $\min$ & $25 \%$ & $50 \%$ & $75 \%$ & $\max$ \\
\hline 1994 & 4213.51 & 4005.48 & 120.00 & 446.51 & 3425.41 & 7459.77 & 19650.00
\end{tabular}

Table 1: The statistical summary of the BTC price datasets from 1 December 2014 to 29 May 2020. 


\subsection{Performance evaluation criteria}

The performance evaluation criteria of regression algorithms for time series forecasting problem are introduced as follows.

\subsubsection{Forecast error (residual forecast error)}

Forecast errors $(\mathrm{FE})$ on a time series forecasting problem are considered as residuals (residual errors). According to Equation 1, the residual error at time $t\left(e^{t}\right)$ can be calculated as the expected outcome $\left(y_{t}\right)$ minus the forecast $\left(f_{t}^{(m)}\right)$ :

$$
e^{t}=\left(y_{t}-f_{t}^{(m)}\right)
$$

$e^{t}$ can be calculated for every observation of the time series. The much more closer the residual error to the zero, the better performance archives.

\subsubsection{Mean forecast error (forecast bias)}

This value is obtained from the average error residual.

$$
\operatorname{BIAS}=\frac{1}{n} \sum_{(i=1)}^{n}\left(e_{i}\right)
$$

Where $\mathrm{n}$ is the number of samples and $e_{i}$ is the ith error. The residual error is either positive or negative. The best BIAS value would be zero.

\subsubsection{Mean absolute error (MAE)}

One of the loss functions that have interesting properties is the mean absolute error which is also called the MAE. The loss function, such as the MSE, uses only the distance between predicted and expected values but does not consider the direction for this difference. The following formula is used to calculate MAE [20]:

$$
\operatorname{MAE}=\frac{1}{\mathrm{n}} \sum_{(\mathrm{i}=1)}^{\mathrm{n}}\left|e_{\mathrm{i}}\right|
$$

When MAE is closer to zero; the ideal model is achieved. 


\subsubsection{Mean squared error (MSE)}

In mean square errors or MSE, the values are positive. It also has a more significant impact on large errors. The loss function computes the mean square error between the predicted and expected values. The Equation 4 shows MSE formula [20]:

$$
\operatorname{MSE}=\frac{1}{n} \sum_{(i=1)}^{n}\left(e_{i}^{2}\right)
$$

\subsubsection{Root mean squared error (RMSE)}

If the effect of the MSE is derived, another loss function is constructed as the "square root of error" which is briefly shown with RMSE.

$$
\operatorname{RMSE}=\sqrt{\frac{1}{n} \sum_{(i=1)}^{n}\left(e_{i}^{2}\right)}
$$

RMSE zero value means the model is actually error-free.

Where $\mathrm{N}$ is the total number of observations, $\left(e_{i}^{2}\right)$ is the actual value.

\subsection{Forecast models}

Figure 1 shows the steps of the current research in which the Box-Jenkins method, ACF, PACF, ARIMA, MA, AR, and Grid Search are used.

\subsubsection{Box-Jenkins method}

The Box-Jenkins method consists of three essential steps. The first step is Model Identification/selection; the second one is Parameter estimation and the last one Statistical model checking. In the identification phase, one or more time series models will be selected by their graphs. The parameters of the selected model are determined in the identification phase. In the control phase (verification), statistical tests are performed to verify the selected model. These tests include the independence of model error values. If the control phase is not approved, then back to the identification phase and selects a new model, the above steps are repeated. 


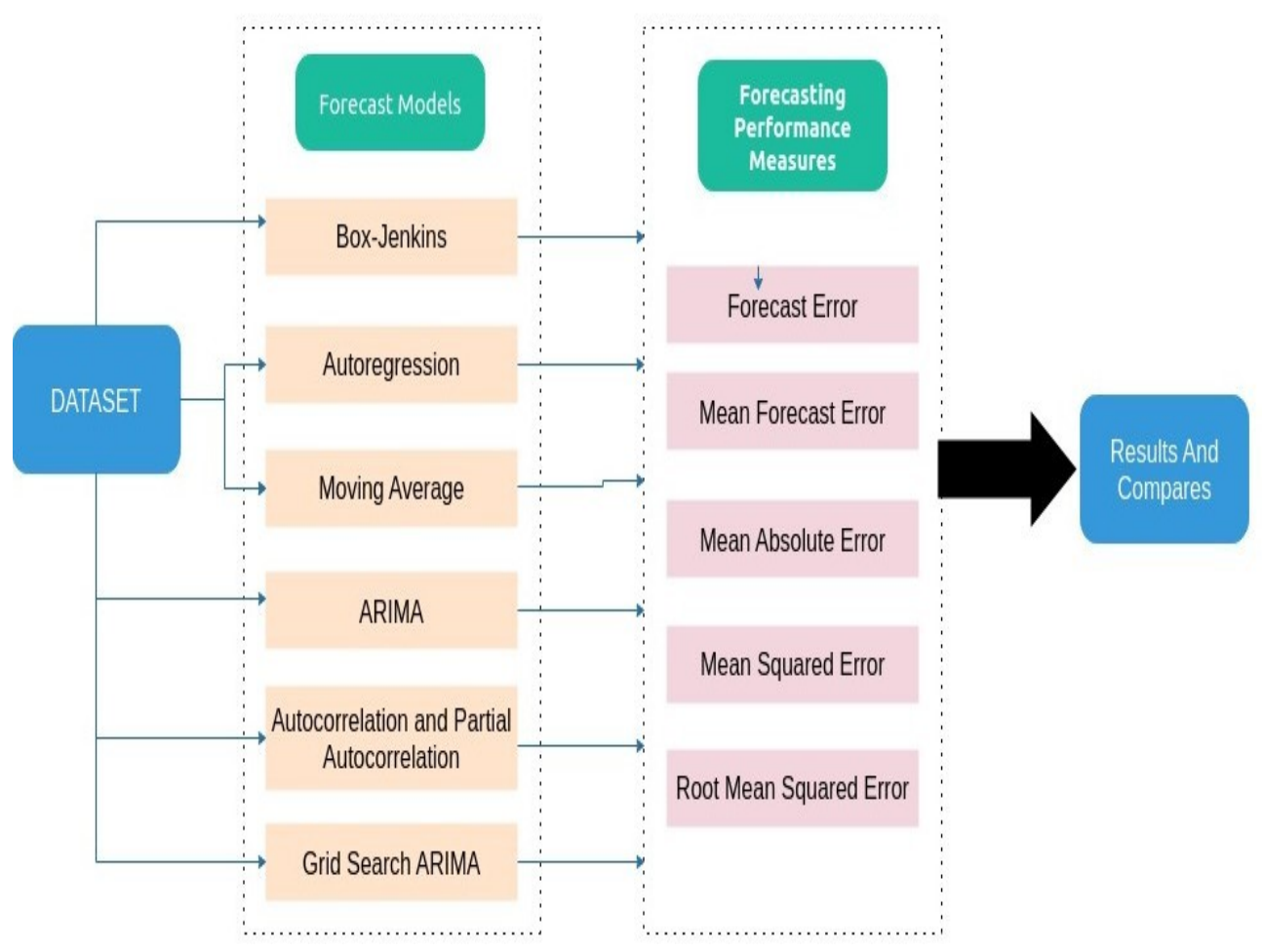

Figure 1: The workflow steps in our proposed model

\subsubsection{Autoregression method}

The Autoregressive (AR) model is used for stationary time series values depending on their previous values. In this case, we consider the number of past observations to predict a value. Therefore, it can be written as follows [37]:

$$
x_{\mathrm{t}}=\mathrm{c}+\Sigma_{(\mathrm{i}=1)}^{\mathrm{P}} \phi_{\mathrm{i}} \mathrm{x}_{(\mathrm{t}-\mathrm{i})}+\epsilon_{\mathrm{t}}
$$

Where $x_{t}$ is the stationary variable, $c$ is constant, the terms in $\phi_{i}$ are autocorrelation coefficients at lags $1,2, p$ and $\epsilon_{\mathrm{t}}$.

\subsubsection{Moving average algorithm}

The moving average (MA) model is written as a linear combination, similar to the AR model, but it is written in terms of a linear combination of errors in 
terms of time. In this case, we consider $q$ the number of previous observations. The relationship between static time-series values in this model is formulated in Equation 7 [37]:

$$
x_{t}=\mu+\Sigma_{(i=0)}^{q} \phi_{i} \epsilon_{t}-i
$$

Where $\mu$ is the expectation of $x_{t}$ (usually assumed equal to zero), the $\phi_{i}$ terms are the weights applied to the current Where $\mu$ is the expectation of $x_{t}$ (usually assumed equal to zero), the $\phi_{i}$ terms are the weights applied to the current.

\subsubsection{ARIMA method}

The autoregressive moving average is an ARIMA (Autoregressive Integrated Moving Average) model, which is a more general model of ARMA. According to the Equation 8, ARIMA combines the combination of two methods of AR and MA [37].

$$
x_{\mathrm{t}}=\mathrm{c}+\Sigma_{(\mathrm{i}=1)}^{\mathrm{P}} \phi_{\mathrm{i}} x_{(\mathrm{t}-\mathrm{i})}+\epsilon_{\mathrm{t}}+\Sigma_{(\mathrm{i}=0)}^{\mathrm{q}} \phi_{\mathrm{i}} \epsilon_{\mathrm{t}}-\mathrm{i}
$$

An ARIMA model has coordinates (p, d, q):

- The $\mathrm{p}$ is defined as the total number of autoregressive terms. In other words, $\mathrm{p}$ is the number of previous observations (from past values) that are used to predict future values. For example, if the value $\mathrm{p}$ is equal to 2 , this means that two previous temporal observations are used in the series to do forecast the future trends.

- $\mathrm{d}$ points to the number of differences which are required to have a stationary the time series (i.e. one with a constant mean, variance, and autocorrelation). For example, if $\mathrm{d}=1$, then the first difference of the time series must be calculated to convert it to the stationary series should be calculated represents the "moving average" error in the previous prediction error in the model or the residual value.

- q represents the MA of the previous FE in the model (the lagged values of the error term). For example, if $q$ has a high value, there is a lagged value of the error term in the model. 


\subsubsection{Autocorrelation and partial autocorrelation methods}

Correlation and partial correlation diagrams are enormously used in time series analysis and forecasting. These are diagrams that summarize the strength of the relationship with observations in a time series with observations at the previous stages. Partial Autocorrelation and Autocorrelation index are used to determine whether the data are stationary or not? The autocorrelation and autocorrelation index for "different degrees" measures the correlation coefficient (CC) between the series and the delay of variables over time. A process is achieved when the time series follows a particular pattern in which the present value depends on the previous values.

\subsubsection{Grid search method}

Grid search (GS) is a nifty approach that tries to explore data space exhaustively using a manually specified hyperparameter subset of the search space for a selected algorithm while Random search choses values for all hyperparameters independently based on their probability distributions. Accurate tuning (Fine Tuning) means finding the best parameter for machine learning algorithms to improve the results. An optimized planning is an effective practical step that can lead to noticeable improvements at the output of the ARIMA method. The optimal parameters can be automatically found on the Grid Search.

\section{Experimental results}

As noted, the data set includes daily BTC close price from 1 December 2014 to 29 May 2020 [44]. In the simulation, we have used $70 \%$ of data as training data and $30 \%$ as the test data. We have used Python programing language and related libraries for time series to implement our simulations.

In time series, it is essential to evaluate and compare the results to spot the best predictor with a minimum error. In our study, the results obtained are compared using BIAS, MAE, MSE, and RMSE. In the term of error, a zero error indicates complete skill for prediction. In Tables 2 and 3, the obtained results are compared well. Figures 2 to 5 are derived from Table 2 and demonstrate the results separately for each algorithm.

In Figures 6, 7, 8, and 9, autocorrelation functions (ACF) and partial autocorrelation (PACF) are calculated for the AR, MA, ARIMA, and Box Jenkins

algorithms. We know these functions reveal the logical link between data in 


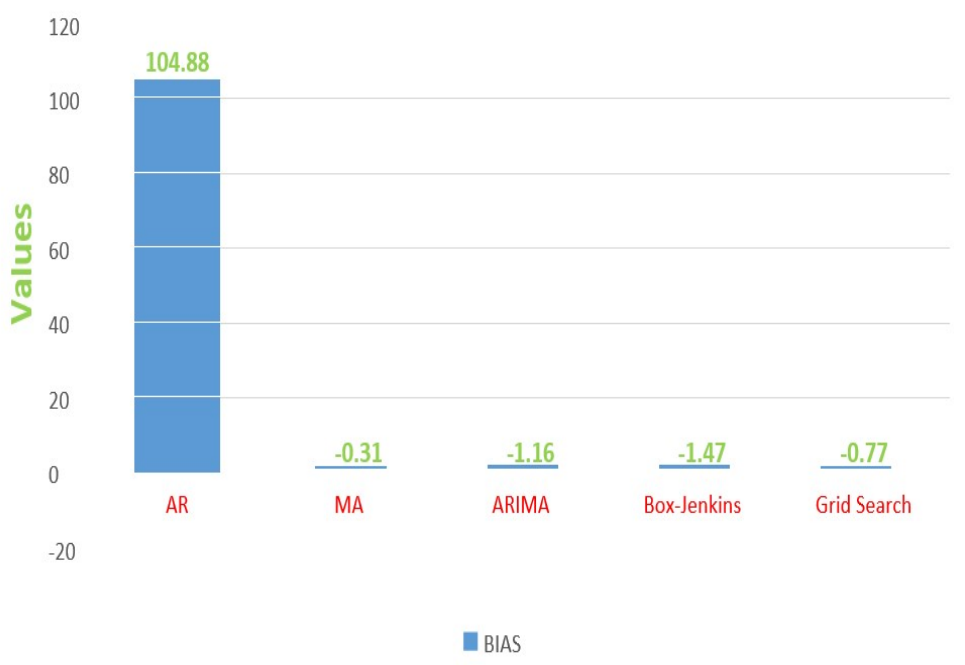

Figure 2: Bias comparison for all algorithms

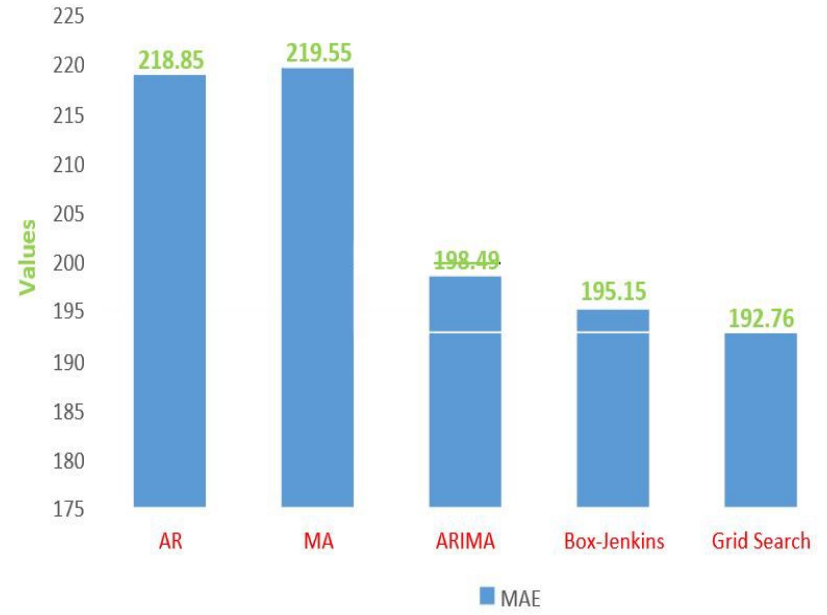

Figure 3: MAE comparison for all algorithms 


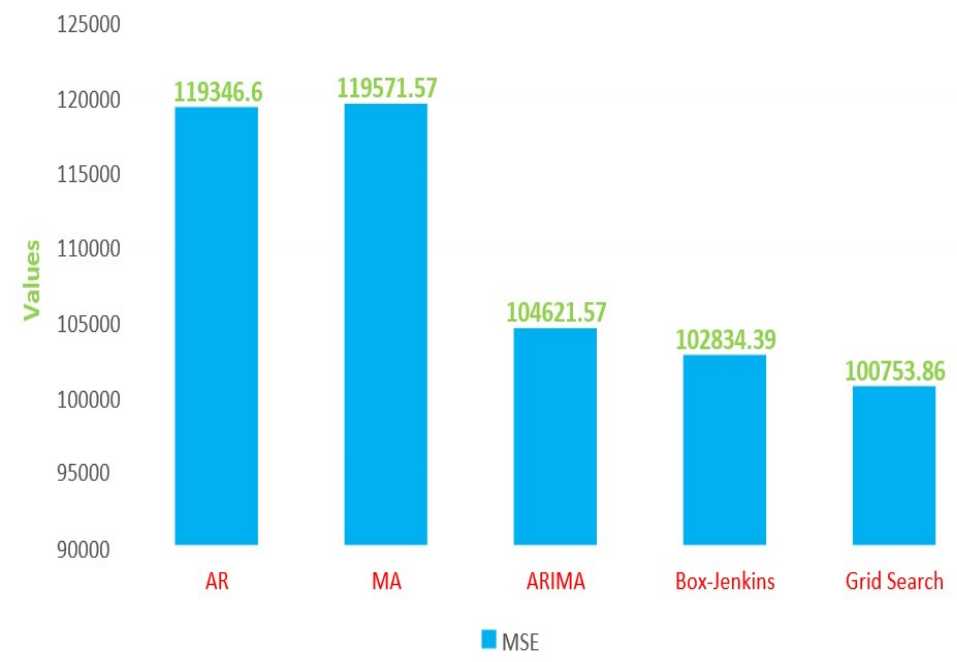

Figure 4: MSE comparison for all algorithms

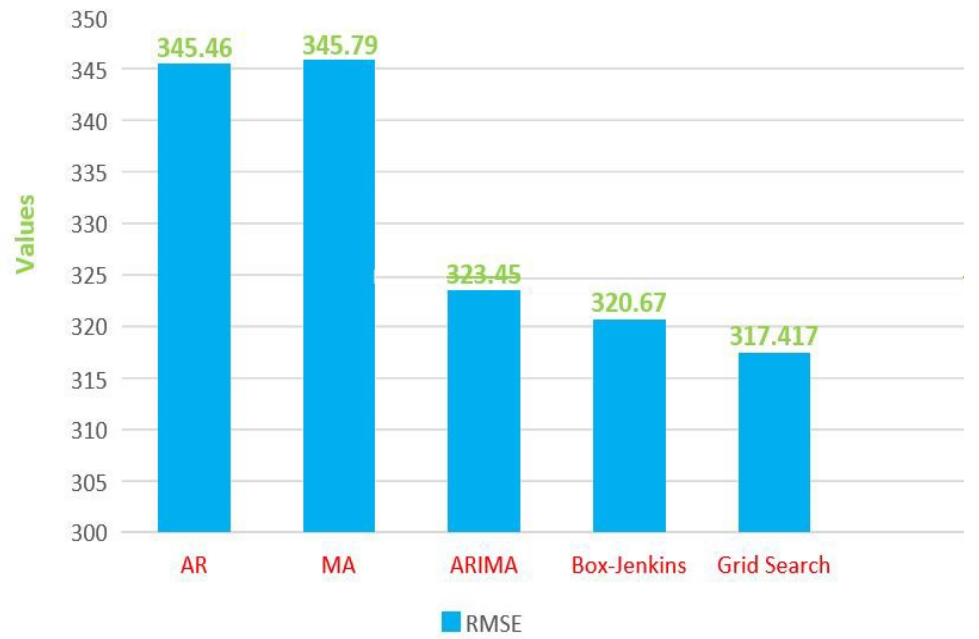

Figure 5: RMSE comparison for all algorithms 


\begin{tabular}{c|c|c|c|c}
\hline Forecast methods & RMSE & MSE & MAE & MFE \\
\hline AR & 345.46 & 119346.60 & 218.85 & 104.88 \\
\hline MA & 345.79 & 119571.57 & 219.55 & -0.31 \\
\hline ARIMA & 323.45 & 104621.57 & 198.49 & -1.16 \\
\hline Box-Jenkins & 320.67 & 102834.39 & 95.15 & -1.47 \\
\hline Grid Search & 317.417 & 100753.86 & 192.76 & -0.77 \\
\hline
\end{tabular}

Table 2: Different evaluation methods for various prediction algorithms.

\begin{tabular}{c|c}
\hline AR & $-425.71,148.50,-112.42, \ldots \ldots \ldots 315.33,396.05,89.32$ \\
\hline MA & $-435.46,153.67,-124.88, \ldots \ldots \ldots 294.43,388.70,67.43$ \\
\hline ARIMA & $-351.55,108.85,-191.76, \ldots \ldots \ldots 347.01,362.92,-116.02$ \\
\hline Box- Jenkins & $-345.70,129.47,-150.95, \ldots \ldots \ldots 373.48,381.12,-147.27$ \\
\hline Grid Search & Best ARIMA $(0,2,0), \mathrm{FE}=-527.228$ \\
\hline
\end{tabular}

Table 3: Assessment of forecasting algorithms with the Forecast Error (FE).

time series. We first extracted prediction data based on the mentioned algorithms, and then we applied ACF and PACF. In Figure 6(a), an ACF plot demonstrates correlation coefficients (CC) bar chart of a time series and lagged values for the AR model. In Figure 6(b), a PACF plot indicates the partial correlation between the series and lags of itself. For the AR process, it can be seen that the ACF plot decreases gradually while simultaneously the PACF has a severe drop after p significant lags.

Figure 7 show ACF and PACF for a MA process. In this figure, the ACF fall down sharply after a $q$ number of lags while PACF follows a gradual declining pattern.

As mentioned before, a model that can get lower errors represents the best model. In our research the Grid Search method is the only algorithm that led to the optimal values in most performance metrics such as MAE equal to 192.76, RMSE $=317.417$, and MSE equal to 100753.86.

Ultimately, we compare our model with some available methods in Table 4 and Table 5. Table 4 indicates that our model outperforms others in term of MSE while in Table 5, it shows better achievement based on RMSE and MAE.

\begin{tabular}{c|c|c|c|c}
\hline Performance metric & The proposed & {$[20]$} & {$[1]$} & {$[16]$} \\
\hline MSE & 104621.57 & 170962.195 & $21,215,311$ & 2519603.08 \\
\hline
\end{tabular}

Table 4: Comparing BTC price prediction algorithms based on the MSE. 


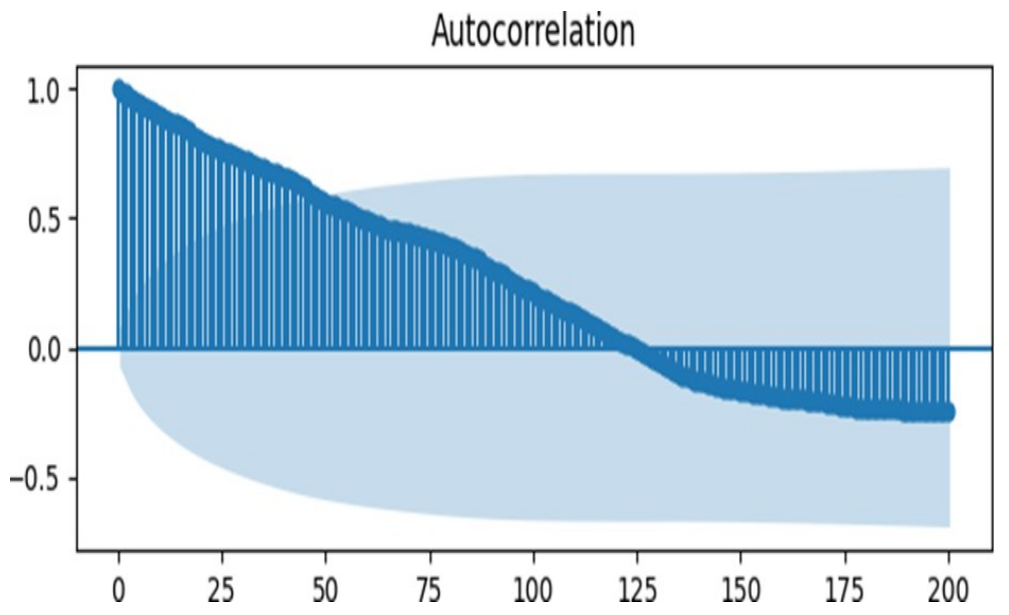

(a)

Partial Autocorrelation

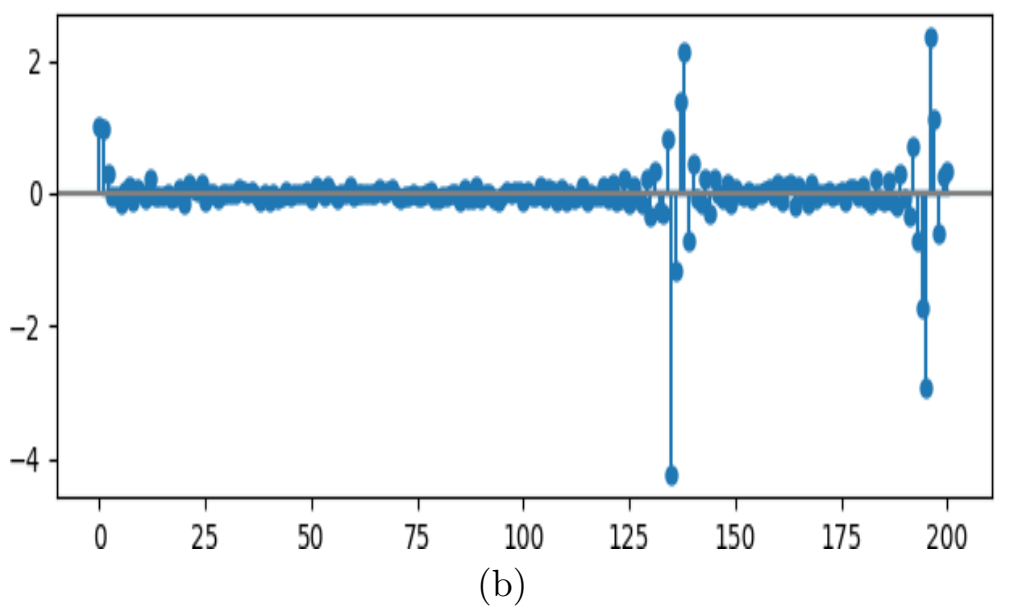

Figure 6: Results of the ACF and PACF methods for the predicted values by the AR method.

(a) ACF plots of the Prediction from AR models

(b) PACF plots of the Prediction from AR models 


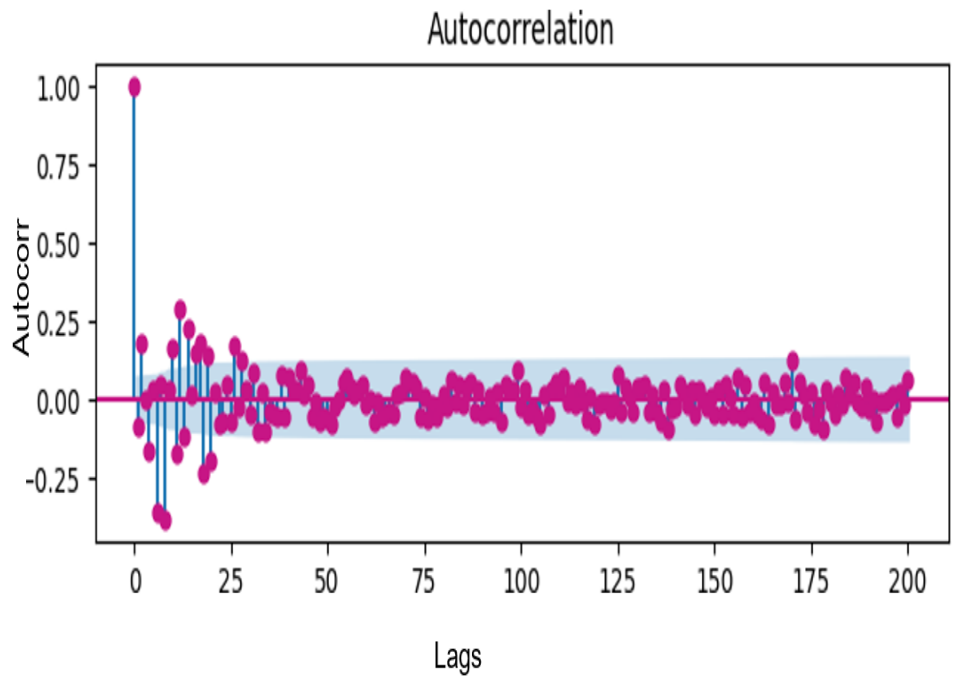

(a)

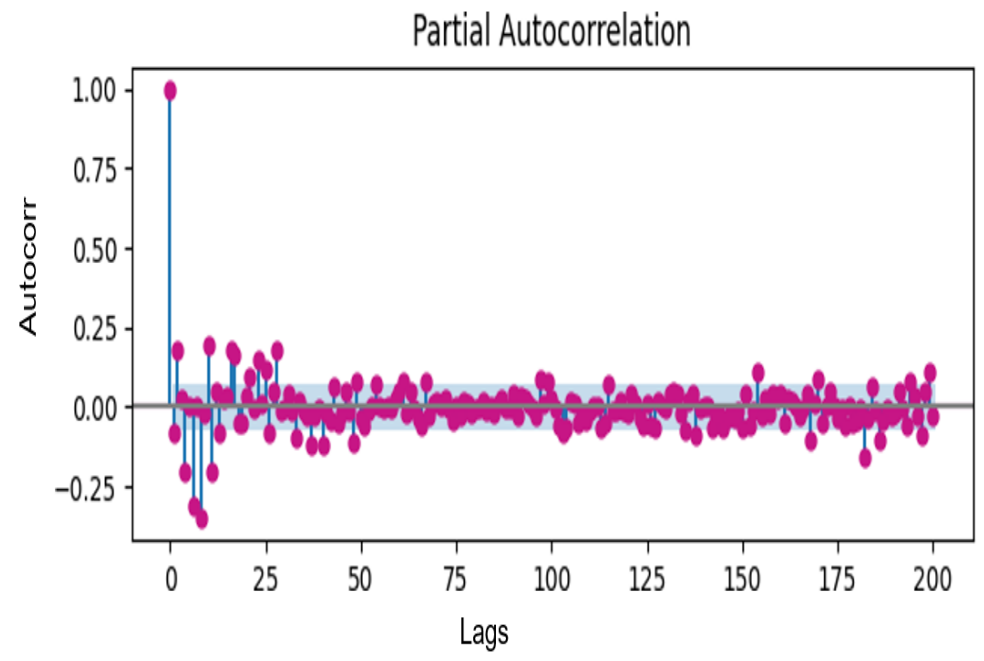

(b)

Figure 7: Results of the ACF and PACF methods for the predicted values by the MA method.
(a) ACF plots of the Prediction from MA models
(b) PACF plots of the Prediction from MA models 


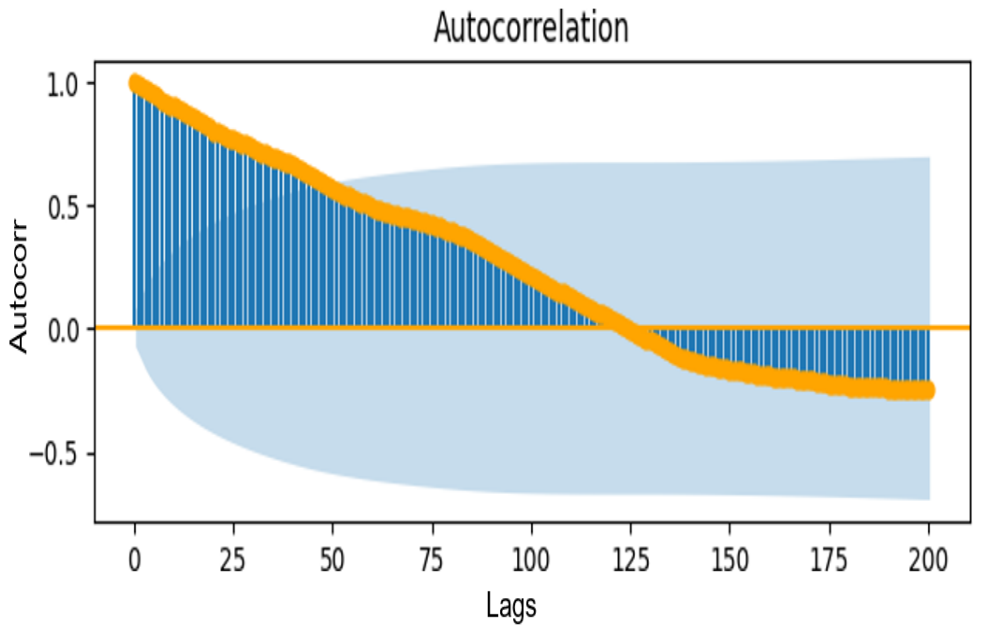

(a)

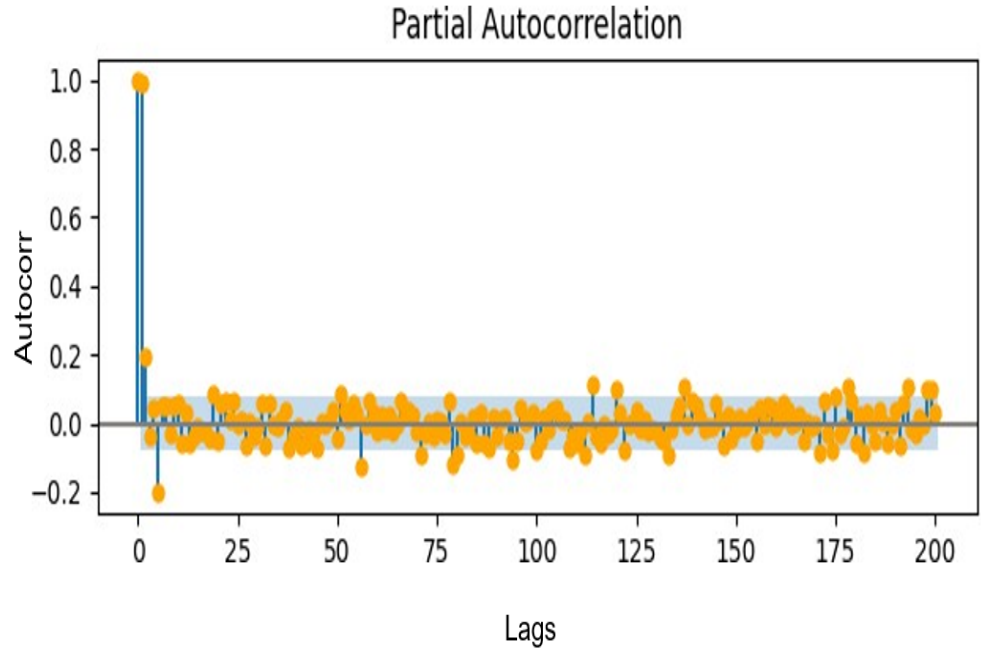

(b)

Figure 8: Results of the ACF and PACF method for ACF plots of the Prediction from ARIMA.
(a) ACF plots of the Prediction from ARIMA models
(b) PACF plots of the Prediction from ARIMA models 


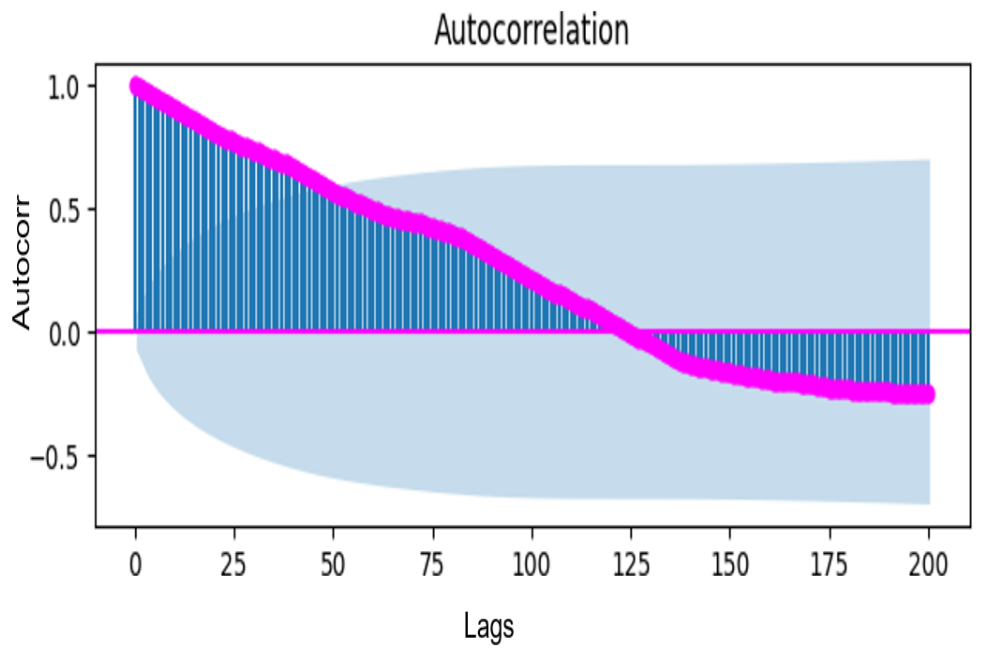

(a)

Partial Autocorrelation

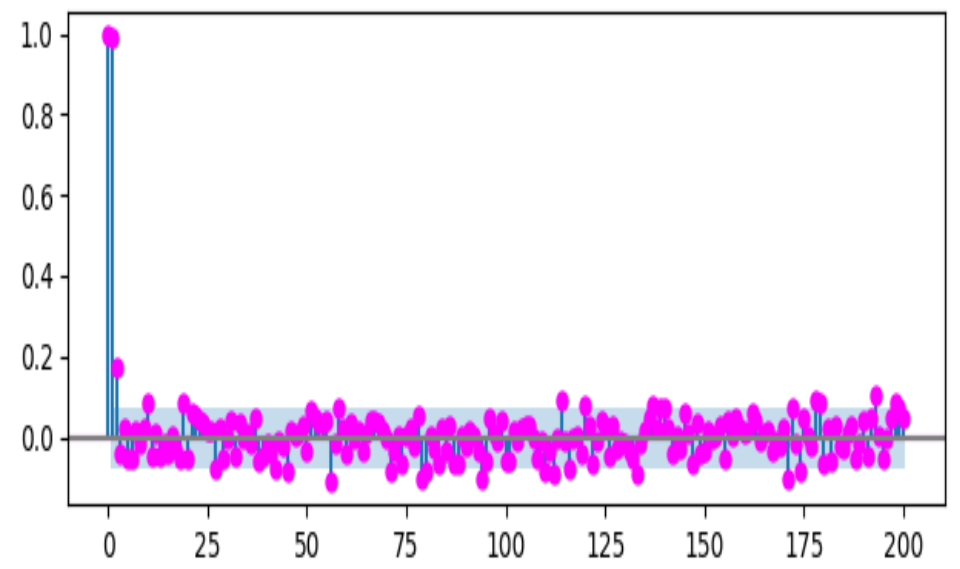

(b)

Figure 9: Results of the ACF and PACF method results for predicted values predicted by the Box-Jenkins method.

(a) ACF plots (b) PACF plots 


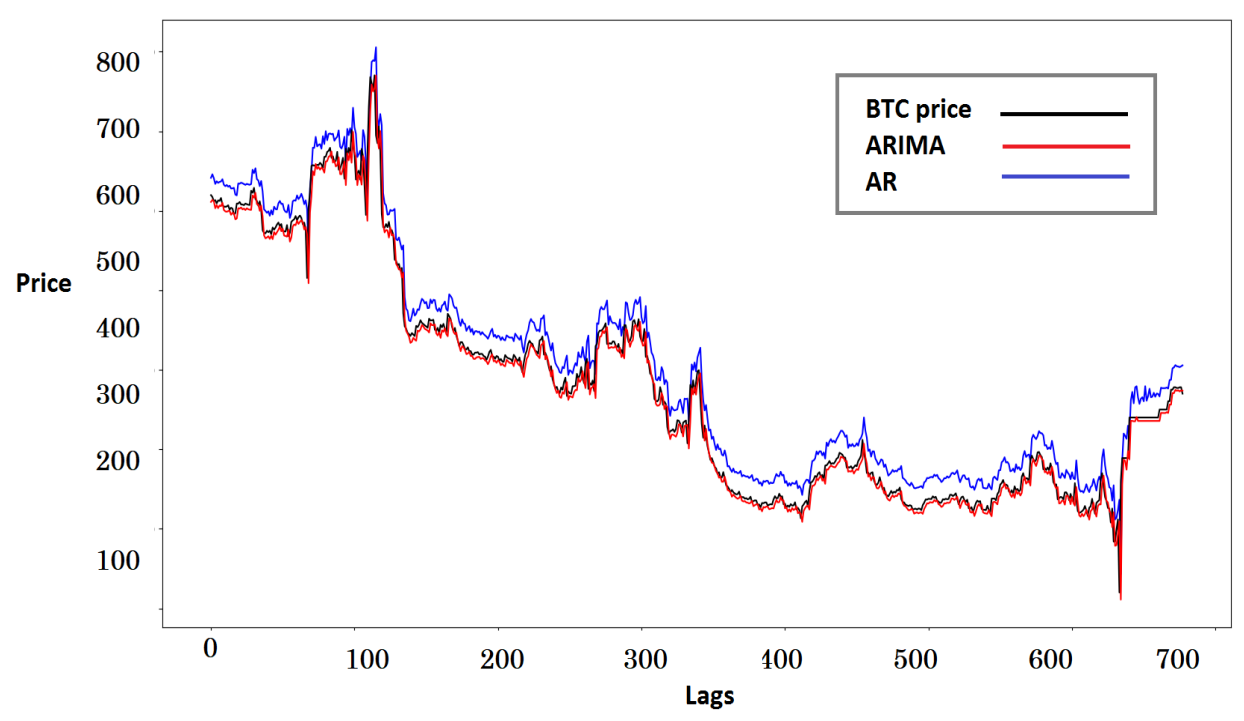

Figure 10: Comparison of ARIMA and AR algorithms with the current value of bitcoin price from 1 December 2014 to 29 May 2020 (Current price of Bitcoin (black), ARIMA (red), AR (blue)).

\begin{tabular}{c|c|c}
\hline Performance metric & The proposed & {$[16]$} \\
\hline RMSE & 323.45 & 1587.32 \\
\hline MAE & 198.49 & 920.45 \\
\hline
\end{tabular}

Table 5: Comparison of BTC price prediction models using the RMSE and MAE.

In Figure 10, a comparison among real price of bitcoin and two prediction models is depicted. In this figure, our proposed algorithm based on grid search ARIMA (red line) greedily tries to track the real BTC price (black line) and it outperforms other models based on AR.

Finally, we have extended our dataset to cover forward test. In the early version, the BTC price datasets from 1 December 2014 to 29 May 2020 was used in training and validation steps. In the new version, we added some new daily price samples from 1 June to 30 June (2020) as unseen data. The unseen data has fitted to the model in order to evaluate the forward testing results. 


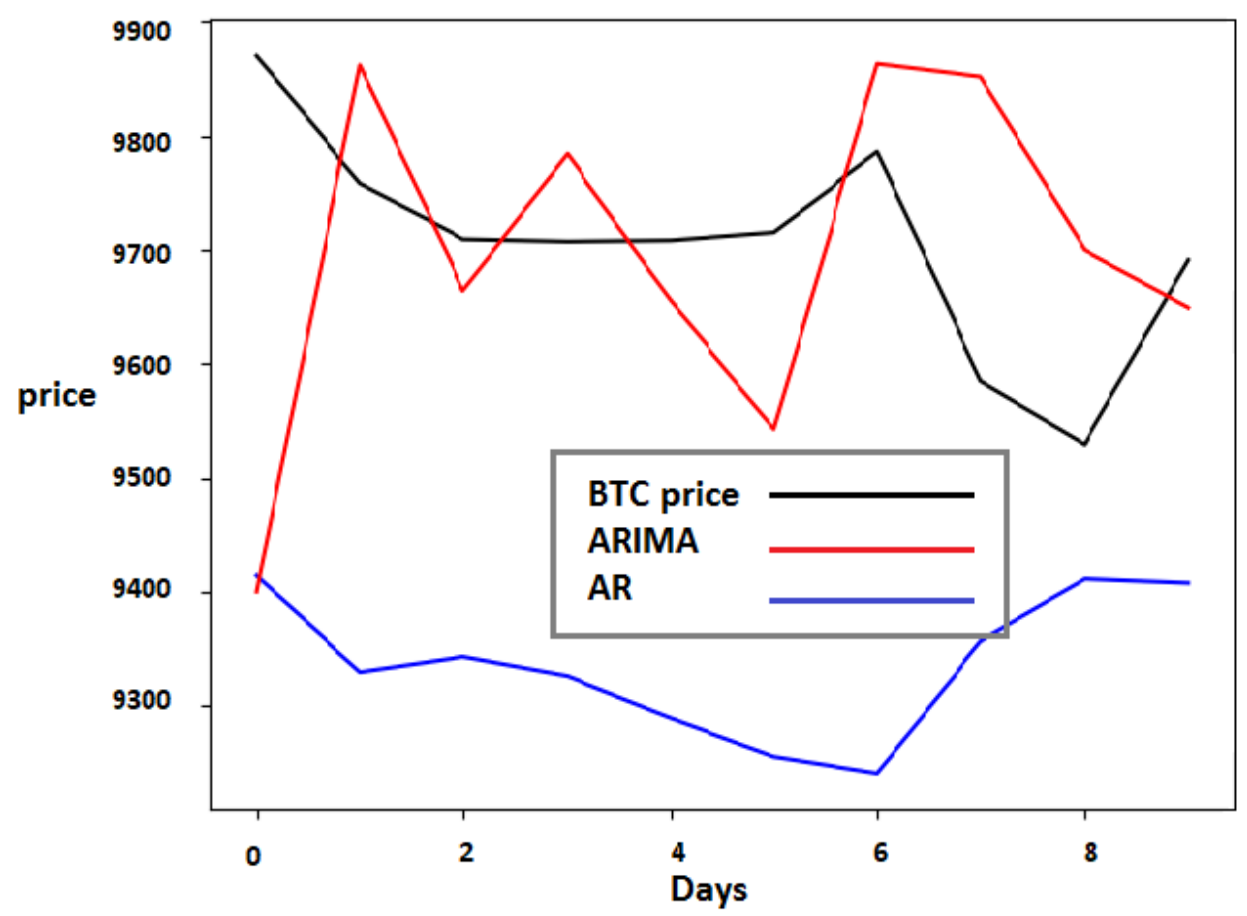

Figure 11: Comparison of ARIMA and AR algorithms with the current value of bitcoin price for June 2020 (Current price of Bitcoin (black), ARIMA (red), AR (blue)).

In Figure 11, it can be seen that the accuracy of our algorithm is much higher even for unseen data.

\section{Conclusions and future work}

As an emerging digital currency, Bitcoin has got much attention nowadays. In fact, BTC is the most valuable encrypted currency globally traded in almost all cryptocurrency exchanges. It provides a fantastic opportunity to make price prediction due to its relatively low-maturity technology with very high price volatility. Developing an accurate and automated predictive system for BTC with non-linear and high range variations is still an open challenging task.

In our study, we have evaluated the number of algorithms using several evaluation criteria. In summary, we have used the Box-Jenkins model, Au- 
toregression, Moving Average, ARIMA model, Autocorrelation and Partial Autocorrelation model, and Grid Search model. Besides, to evaluate and compare them, we used FE, MFE, MAE, MSE, and RMSE. In our study, the Grid Search method has the best performance with lower errors than other methods. This method tries to find the best results with optimizing the ARIMA method. It achieved the minimum value in the MAE at 192.76, the MSE equal to 100753.86 , and RMSE $=317.417$. Furthermore, the values of the forecast error method in the Grid Search have the lowest values approximately close to zero. Consequently, the proposed model outperformed others in most performance metrics.

As both the test and train data are scattered randomly in the entire timeinterval, the problem actually would be one of price interpolation or filling the gaps problems (not extrapolation). The main contribution is a thorough comparison of the performance of existing methods and also optimizing models based on Grid search optimization. The Grid searching is the process of scanning the data to configure optimal parameters for a given model. Based on the Grid search, the optimal hyperparameters of our model have tunned. As a result, the more accurate predictions have achieved in our study.

Price volatility has been extensively investigated on financial markets, but due to the recent emergence of Bitcoin market, researchers have started to scratch the surface in this area. Hence, the excessive volatility of Bitcoin and how determine it properly has not yet been sufficiently studied providing for an extensive research gap. Consequently, similar to the most available studies in BTC price prediction, we have not aimed to offer a discussion into Bitcoin price volatility but it may be our new study in the future to solve this issue using deep learning algorithms. Today, deep learning algorithms are deployed widely in various applications $[23,9,2]$. To continue this research, more studies can be done on the non-linear and even non-stationary BTC price time series in lower time scales using deep learning models.

Funding Statement. There is no specific funding for this study.

Conflicts of Interest. The authors declare that they have no conflicts of interest to report regarding the present study. 


\section{References}

[1] G. Abdoli, Comparing the prediction accuracy of LSTM and ARIMA models for time-series with permanent fluctuation, Periódico do Núcleo de Estudos e Pesquisas sobre Gênero e DireitovCentro de Ciências Jurídicas-Universidade Federal da Paraíba, vol. 9, 2020. $\Rightarrow 277$

[2] F. Abedini, M. Bahaghighat, M. S'hoyan, Wind turbine tower detection using feature descriptors and deep learning, Facta Universitatis, Series: Electronics and Energetics, 33, 1 (2019) 133-153. $\Rightarrow 267,284$

[3] R. Ali, J. Barrdear, R. Clews, J. Southgate, Innovations in payment technologies and the emergence of digital currencies, Bank of England Quarterly Bulletin, Q3, 2014. $\Rightarrow 266$

[4] E. Amouee, M. M. Zanjireh, M. Bahaghighat, M. Ghorbani, A new anomalous text detection approach using unsupervised methods, Facta universitatis-series: Electronics and Energetics, 33, 4 (2020) 631-653. $\Rightarrow 267$

[5] G. S. Atsalakis, I. G. Atsalaki, F. Pasiouras, C. Zopounidis, Bitcoin price forecasting with neuro-fuzzy techniques, European Journal of Operational Research, 276, 2 (2019) 770-780. $\Rightarrow 268$

[6] M. K. Bahaghighat, R. Akbari et al., Fingerprint image enhancement using GWT and DMF, in 2010 2nd International Conference on Signal Processing Systems, vol. 1. IEEE, 2010, pp. V1-253-257. $\Rightarrow 268$

[7] M. K. Bahaghighat, J. Mohammadi, Novel approach for baseline detection and Text line segmentation, International Journal of Computer Applications, 51, 2 (2012) 9-16. $\Rightarrow 267$

[8] M. K. Bahaghighat, F. Sahba, E. Tehrani, Textdependent Speaker Recognition by Combination of LBG VQ and DTW for Persian language. International Journal of Computer Applications, 51, 16 (2012) 23-27. $\Rightarrow 267$

[9] M. Bahaghighat, Q. Xin, S. A. Motamedi, M. M. Zanjireh, A. Vacavant, Estimation of wind turbine angular velocity remotely found on video mining and convolutional neural network, Applied Sciences, 10, 10 (2020) 3544. $\Rightarrow 267,284$

[10] M. Bahaghighat, F. Abedini, Q. Xin, M. M. Zanjireh, S. Mirjalili, Using machine learning and computer vision to estimate the angular velocity of wind turbines in smart grids remotely, Energy Reports, 2021. $\Rightarrow 268$

[11] M. Briere, K. Oosterlinck, A. Szafarz, Virtual currency, tangible return: Portfolio diversification with bitcoin, Journal of Asset Management, 16, 6 (2015) 365-373. $\Rightarrow 267$

[12] G. M. Caporale, L. Gil-Alana, A. Plastun, Persistence in the cryptocurrency market, Research in International Business and Finance, 46 (2018) 141-148. $\Rightarrow$ 266

[13] G. Caffyn, What is the bitcoin block size debate and why does it matter, http://www.coindesk.com/, 2015. $\Rightarrow 266$

[14] M. J. Casey, P. Vigna, Bitcoin and the digital-currency revolution, The Wall Street Journal, Jan. 23, 2015. $\Rightarrow 266$ 
[15] C. Chatfield, M. Yar, Holt-Winters forecasting: some practical issues, Journal of the Royal Statistical Society: Series D (The Statistician), 37, 2 (1988) 129-140. $\Rightarrow 267$

[16] A. Chaudhari, Forecasting Cryptocurrency Prices using Machine Learning, 2020, Dublin, National College of Ireland, Ph.D. dissertation. $\Rightarrow 277,282$

[17] Z. Chen, C. Li, W. Sun, Bitcoin price prediction using machine learning: An approach to sample dimension engineering, Journal of Computational and Applied Mathematics, 365 (2020) p. $112395 . \Rightarrow 269$

[18] P. Ciaian, M. Rajcaniova, D. Kancs, The economics of BitCoin price formation, Applied Economics, 48, 19 (20160) 1799-1815. $\Rightarrow 266$

[19] J. Debler, Foreign initial coin offering issuers beware: the Securities and Exchange Commission is watching, Cornell Int'l LJ, 51 (2018) 245-245. $\Rightarrow 266$

[20] J. Fiaidhi, A. Sabah, M. A. Ansari, Z. Ayaz, Bitcoin Price Prediction using ARIMA Model, 2020. $\Rightarrow 270,271,277$

[21] N. Gandal, H. Halaburda, Competition in the cryptocurrency market, 2014. $\Rightarrow$ 266

[22] N. Gandal, H. Halaburda, Can we predict the winner in a market with network effects? Competition in cryptocurrency market, Games, 7, 3 (2016) 16. $\Rightarrow 266$

[23] M. Ghorbani, M. Bahaghighat, Q. Xin, F. Özen, ConvLSTMConv network: a deep learning approach for sentiment analysis in cloud computing, Journal of Cloud Computing, 9, 1 (2020) 1-12. $\Rightarrow 267,284$

[24] P. Giudici, I. Abu-Hashish, What determines bitcoin exchange prices? A network VAR approach, Finance Research Letters, 28 (2019) 309-318. $\Rightarrow 268$

[25] A. Hajikarimi, M. Bahaghighat, Optimum Outlier Detection in Internet of Things Industries Using Autoencoder, in Frontiers in Nature-Inspired Industrial Optimization. Springer, 2022, pp. $77-92 . \Rightarrow 268$

[26] S. Hasani, M. Bahaghighat, M. Mirfatahia, The mediating effect of the brand on the relationship between social network marketing and consumer behavior, Acta Technica Napocensis, 60, 2 (2019) 1-6. $\Rightarrow 267$

[27] G. Hileman, M. Rauchs, Global cryptocurrency benchmarking study, Cambridge Centre for Alternative Finance, 33 (2017) 33-113. $\Rightarrow 266$

[28] I. Kaastra, M. Boyd, Designing a neural network for forecasting financial and economic time series, Neurocomputing, 10, 3 (1996) 215-236. $\Rightarrow 267$

[29] P. Katsiampa, Volatility estimation for Bitcoin: A comparison of GARCH models, Economics Letters, 158 (2017) 3-6. $\Rightarrow 268$

[30] M. Lischke, B. Fabian, Analyzing the bitcoin network: The first four years, Future Internet, 8, 1 (2016) $7 . \Rightarrow 266$

[31] M. Mudassir, S. Bennbaia, D. Unal, M. Hammoudeh, Time-series forecasting of Bitcoin prices using high-dimensional features: a machine learning approach, Neural Computing and Applications, pp. 1-15, 2020. $\Rightarrow 268$

[32] K. Rathan, S. V. Sai, T. S. Manikanta, Crypto-currency price prediction using decision tree and regression techniques, in 2019 3rd International Conference on Trends in Electronics and Informatics (ICOEI). IEEE, 2019, pp. 190-194. $\Rightarrow$ 267 
[33] F. Reid, M. Harrigan, An analysis of anonymity in the bitcoin system, in Security and privacy in social networks. Springer, 2013, pp. 197-223. $\Rightarrow 266$

[34] D. Ron, A. Shamir, Quantitative analysis of the full bitcoin transaction graph, in International Conference on Financial Cryptography and Data Security. Springer, 2013, pp. 6-24. $\Rightarrow 266$

[35] M. S. S. Sajadi, M. Babaie, M. Bahaghighat, Design and implementation of fuzzy supervisor controller on optimized DC machine driver, in 2018 8th Conference of AI 8 Robotics and 10th RoboCup Iranopen International Symposium (IRANOPEN). IEEE, 2018, pp. 26-31. $\Rightarrow 268$

[36] A. Shamseen, M. M. Zanjireh, M. Bahaghighat, Q. Xin, Developing a parallel classifier for mining in big data sets, IIUM Engineering Journal, 22, 2 (2021) $119-134 . \Rightarrow 268$

[37] S. Siami-Namini, N. Tavakoli, A. S. Namin, A comparison of ARIMA and LSTM in forecasting time series, pp. 1394-1401, 2018. $\Rightarrow 272,273$

[38] S. Trimborn, W. K. Härdle, CRIX an Index for blockchain based Currencies, Journal of Empirical Finance, 49 (2018) 107-222. $\Rightarrow 266$

[39] C. Trucíos, Forecasting Bitcoin risk measures: A robust approach, International Journal of Forecasting, 35, 3 (2019) 836-847. $\Rightarrow 268$

[40] P. Vigna, M. J. Casey, The age of cryptocurrency: how bitcoin and the blockchain are challenging the global economic order. Macmillan, 2016. $\Rightarrow 266$

[41] H. White, Economic prediction using neural networks: The case of IBM daily stock returns, in ICNN, vol. 2, 1988, pp. 451-458. $\Rightarrow 267$

[42] L. H. White, The market for cryptocurrencies, Cato J. 35 (2015) 383. $\Rightarrow 266$

[43] D. Yermack, Is Bitcoin a real currency? An economic appraisal (No. w19747), National Bureau of Economic Research, 36, 2 (2013) 843-850, $\Rightarrow 266$

[44] $* * *$, Historical OHLC price data includes volume, www.cryptodatadownload.com $\Rightarrow 269,274$ 